\title{
Simple homotopy types and finite spaces
}

\author{
Jonathan Ariel Barmak, Elias Gabriel Minian* \\ Departamento de Matemática, FCEyN, Universidad de Buenos Aires, Buenos Aires, Argentina \\ Received 29 November 2006; accepted 28 November 2007 \\ Available online 3 January 2008 \\ Communicated by Mark Hovey
}

\begin{abstract}
We present a new approach to simple homotopy theory of polyhedra using finite topological spaces. We define the concept of collapse of a finite space and prove that this new notion corresponds exactly to the concept of a simplicial collapse. More precisely, we show that a collapse $X \searrow Y$ of finite spaces induces a simplicial collapse $\mathcal{K}(X) \searrow \mathcal{K}(Y)$ of their associated simplicial complexes. Moreover, a simplicial collapse $K \searrow L$ induces a collapse $\mathcal{X}(K) \searrow \mathcal{X}(L)$ of the associated finite spaces. This establishes a one-to-one correspondence between simple homotopy types of finite simplicial complexes and simple equivalence classes of finite spaces. We also prove a similar result for maps: We give a complete characterization of the class of maps between finite spaces which induce simple homotopy equivalences between the associated polyhedra. This class describes all maps coming from simple homotopy equivalences at the level of complexes. The advantage of this theory is that the elementary move of finite spaces is much simpler than the elementary move of simplicial complexes: It consists of removing (or adding) just a single point of the space.

(c) 2007 Elsevier Inc. All rights reserved.
\end{abstract}

MSC: 57Q10; 55U05; 57N65; 55P15; $06 \mathrm{~A} 06$

Keywords: Finite spaces; Simplicial complexes; Simple homotopy types; Posets; Weak homotopy equivalences; Simple homotopy equivalences

\footnotetext{
* Corresponding author.

E-mail addresses: jbarmak@dm.uba.ar (J.A. Barmak), gminian@dm.uba.ar (E.G. Minian).
} 


\section{Introduction}

J.H.C. Whitehead's theory of simple homotopy types is inspired by Tietze's theorem in combinatorial group theory, which states that any finite presentation of a group could be deformed into any other by a finite sequence of elementary moves, which are now called Tietze transformations. Whitehead translated these algebraic moves into the well-known geometric moves of elementary collapses and expansions of finite simplicial complexes. His beautiful theory turned out to be fundamental for the development of piecewise-linear topology: The s-cobordism theorem, Zeeman's conjecture [18], the applications of the theory in surgery, Milnor's classical paper on Whitehead Torsion [10] and the topological invariance of torsion are some of its major uses and advances.

In this paper we show how to use finite topological spaces to study simple homotopy types. There is a strong relationship between finite spaces and finite simplicial complexes, which was discovered by McCord [9]. Explicitly, given a finite simplicial complex $K$, one can associate to $K$ a finite $T_{0}$-space $\mathcal{X}(K)$ which corresponds to the poset of simplices of $K$ ordered by inclusion. Moreover, a simplicial map $\varphi: K \rightarrow L$ gives rise to a continuous map $\mathcal{X}(\varphi)$ between the associated finite spaces. Conversely, one can associate to a finite $T_{0}$-space $X$ a simplicial complex $\mathcal{K}(X)$, whose simplices are the non-empty chains of $X$, and a weak homotopy equivalence $\mathcal{K}(X) \rightarrow X$. This construction is also functorial.

In [2] we showed that finite spaces are very useful for studying homotopy invariants of (general) spaces. In fact, in that article we were looking for minimal finite models of some spaces, i.e. the smallest finite spaces which are weak (homotopy) equivalent to a given space. Finite spaces are closely related to finite posets, which have become an important tool in algebraic and geometric topology (see for example Quillen's paper [12], Björner's paper [3] and Björner, Wachs and Welker's [4]). The finite space point of view adds a new dimension to finite posets and allows the development of new and more appropriate techniques based on the combinatorics and the topology of these objects.

It is easy to prove that if two finite $T_{0}$-spaces $X, Y$ are homotopy equivalent, their associated simplicial complexes $\mathcal{K}(X), \mathcal{K}(Y)$ are also homotopy equivalent. Furthermore, Osaki [11] showed that in this case, the latter have the same simple homotopy type. Nevertheless, we noticed that the converse of this result is not true in general: There are finite spaces with different homotopy types whose associated simplicial complexes have the same simple homotopy type. Starting from this point, we were looking for the relation that $X$ and $Y$ should satisfy for their associated complexes to be simple homotopy equivalent. More specifically, we wanted to find an elementary move in the setting of finite spaces (if it existed) which corresponds exactly to a simplicial collapse of the associated polyhedra.

We discovered this elementary move when we were looking for a homotopically trivial finite space (i.e. weak equivalent to a point) which was non-contractible. In order to construct such a space, we developed a method of reduction, i.e. a method that allows us to reduce a finite space to a smaller weak equivalent space. This method of reduction together with the homotopically trivial and non-contractible space (of 11 points) that we found are exhibited in Section 3. Surprisingly, this method, which consists of removing a weak point of the space (see Definition 3.2), turned out to be the key to solve the problem of translating simplicial collapses into this setting.

We will say that two finite spaces are simply equivalent if we can obtain one of them from the other by adding and removing weak points. If $Y$ is obtained from $X$ by only removing weak points, we say that $X$ collapses to $Y$ and write $X \searrow Y$. The first main result of this article is the following 


\section{Theorem 3.10.}

(a) Let $X$ and $Y$ be finite $T_{0}$-spaces. Then, $X$ and $Y$ are simply equivalent if and only if $\mathcal{K}(X)$ and $\mathcal{K}(Y)$ have the same simple homotopy type. Moreover, if $X \searrow Y$ then $\mathcal{K}(X) \searrow \mathcal{K}(Y)$.

(b) Let $K$ and $L$ be finite simplicial complexes. Then, $K$ and $L$ are simple homotopy equivalent if and only if $\mathcal{X}(K)$ and $\mathcal{X}(L)$ are simply equivalent. Moreover, if $K \searrow L$ then $\mathcal{X}(K) \searrow \mathcal{X}(L)$.

In particular, the functors $\mathcal{K}$ and $\mathcal{X}$ induce a one-to-one correspondence between simple equivalence classes of finite spaces and simple homotopy types:

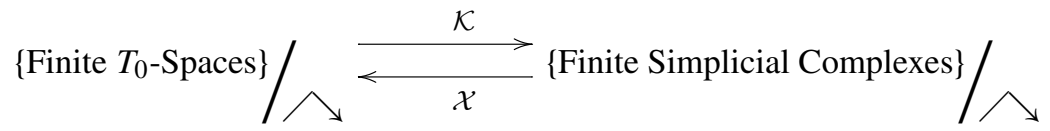

We are now able to study finite spaces using all the machinery of Whitehead's simple homotopy theory for CW-complexes. But also, what is more important, we can use finite spaces to strengthen the classical theory. The elementary move in this setting is much simpler to handle and describe because it consists of adding or removing just one single point.

As an example or application of this theorem, we study collapsible finite spaces and their relationship with collapsible complexes. We also relate simple types of finite spaces with the notion of minimal finite model introduced in [2].

In the last section of this article we investigate the class of maps between finite spaces which induce simple homotopy equivalences between their associated simplicial complexes. To this end, we introduce the notion of a distinguished map. Similarly to the classical case, the class of simple equivalences between finite spaces can be generated, in a certain way, by expansions and a kind of formal homotopy inverses of expansions. Remarkably this class, denoted by $\mathcal{S}$, is also generated by the distinguished maps. The second main result of the article is the following

\section{Theorem 4.13.}

(a) Let $f: X \rightarrow Y$ be a map between finite $T_{0}$-spaces. Then $f$ is a simple equivalence if and only if $\mathcal{K}(f): \mathcal{K}(X) \rightarrow \mathcal{K}(Y)$ is a simple homotopy equivalence.

(b) Let $\varphi: K \rightarrow L$ be a simplicial map between finite simplicial complexes. Then $\varphi$ is a simple homotopy equivalence if and only if $\mathcal{X}(\varphi)$ is a simple equivalence.

\section{Preliminaries}

In this section we recall various results on finite spaces which are needed in Sections 3 and 4. For more details on finite spaces we refer the reader to $[9,14]$ and P. May's notes $[7,8]$.

\subsection{The correspondence between finite spaces and finite posets}

There is a natural relationship between topologies and preorders defined on a finite set $X$. This correspondence, which was studied in first place by Alexandroff [1], can be described as follows. Given a topology $\tau$ on $X$, consider for each point $x$ in $X$, the intersection $U_{x}$ of all open sets containing $x$. This is clearly an open set for each $x$ and the family $B=\left\{U_{x}, x \in X\right\}$ is a basis for the topology $\tau$. This basis is called the minimal basis of $X$ for obvious reasons. Associated 
to $\tau$, there is a preorder structure on $X$ (i.e. a reflexive and transitive relation), defined by $x \leqslant y$ if $x \in U_{y}$. Conversely, if a preorder $\leqslant$ on the finite set $X$ is given, we define for each $x \in X$ the subset $U_{x}=\{y \in X \mid y \leqslant x\}$. It is not hard to see that these subsets form a basis for a topology on $X$, which is the topology associated to the preorder $\leqslant$.

The applications described above define a one-to-one correspondence between topological structures and preorders on $X$. Moreover, the $T_{0}$ separation axiom is equivalent to the antisymmetry of the associated preorder and therefore, $T_{0}$-topologies on $X$ correspond to order relations. Having this equivalence in mind, we will regard finite $T_{0}$-spaces as finite posets and vice versa. We will use both structures according to convenience.

It is very useful to represent finite spaces using Hasse diagrams. The Hasse diagram of a finite $T_{0}$-space $X$ is a digraph whose vertex set is $X$ and whose edges are the ordered pairs $(x, y)$ such that $x<y$ and there exists no $z \in X$ with $x<z<y$.

Example 2.1. Consider the space $X=\{a, b, c, d\}$ whose proper open sets are $\{a, c, d\},\{b, c, d\}$, $\{c, d\}$ and $\{d\}$. Its Hasse diagram is

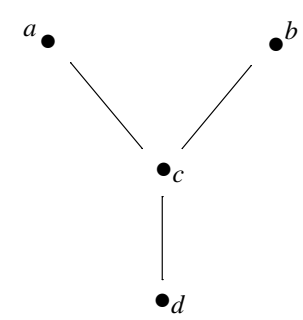

Instead of representing an edge $(x, y)$ with an arrow, one simply writes $y$ over $x$.

Sometimes it is convenient to consider the opposite preorder of a finite space $X$. The space associated to this preorder will be denoted by $X^{\mathrm{op}}$. Concretely, the open sets of $X^{\mathrm{op}}$ are the closed sets of $X$.

Note that a map $f: X \rightarrow Y$ between finite spaces is continuous if and only if it is order preserving. There is also a nice way to describe homotopies. Given two functions $f, g: X \rightarrow Y$, we will say that $f \leqslant g$ if $f(x) \leqslant g(x)$ for every $x \in X$. It is not difficult to prove that if $f$ and $g$ are continuous and $f \leqslant g$, then $f$ is homotopic to $g$ (see [7,14] for more details). In particular, any finite space with maximum or minimum is contractible.

\subsection{Homotopy types}

In 1966 R.E. Stong [14] found a combinatorial way to describe conclusively the homotopy types of finite spaces. He introduced the notions of linear and colinear points and proved that these two kinds of points generate all homotopy equivalences between finite spaces. Essentially, two finite $T_{0}$-spaces $X$ and $Y$ have the same homotopy type if and only if there exists a sequence $X=X_{0}, X_{1}, \ldots, X_{n}=Y$ such that each space is obtained from the previous one by adding or removing a linear or colinear point. Afterwards, Peter May called these points beat points [7].

Following Peter May's language, we will say that a point $x$ of a finite $T_{0}$-space $X$ is an up beat point if the set of points which are greater than $x$ has a minimum. On the other hand, $x \in X$ is said to be a down beat point if the set of points below it has a maximum. This is equivalent to 
say that $x$ is an up beat point of $X^{\mathrm{op}}$. When there is no need to precise if $x$ is an up or a down beat point, we simply say that $x$ is a beat point.

The next obvious remark plays an important role in Theorem 3.10.

Remark 2.2. If $x \in X$ is a beat point, there exists $y \in X, y \neq x$, such that any point which is comparable with $x$ is also comparable with $y$.

It is not difficult to see that if $x \in X$ is a beat point, the inclusion of $X \backslash\{x\}$ in $X$ is a strong deformation retract. Therefore, given a finite $T_{0}$-space $X$, one can remove beat points, one at the time, to obtain a strong deformation retract of $X$ with no beat points. Such a subspace is called a core of $X$. A finite $T_{0}$-space with no beat points is called a minimal finite space.

In [14] Stong proves that every homotopy equivalence between minimal finite spaces is a homeomorphism and therefore, the core of any finite space $X$ is unique up to homeomorphism. It can be described as the smallest space which is homotopy equivalent to $X$. Note that a finite $T_{0}$-space $X$ is contractible if and only if there exists a sequence $X=X_{0} \supsetneq X_{1} \supsetneq \cdots \supsetneq X_{n}=*$, where $X_{i+1}$ is obtained from $X_{i}$ by removing a beat point. Note also that a point $x \in X$ is a beat point if and only if $x$ is a beat point of $X^{\text {op }}$. Therefore, $X$ is contractible if and only if $X^{\text {op }}$ is contractible.

\subsection{Finite spaces and simplicial complexes}

In contrast to Stong's combinatorial approach to homotopy theory of finite spaces, M.C. McCord [9] investigated their relationship with polyhedra. Finite spaces are not in general subspaces of Euclidean spaces. Moreover, they do not have in general the homotopy type of any $T_{1}$ topological space [2]. Nevertheless, their weak homotopy types describe all weak homotopy types of compact polyhedra.

Following McCord [9] (cf. also [8]) one can associate to any finite $T_{0}$-space $X$ a simplicial complex $\mathcal{K}(X)$, whose simplices are the non-empty chains of $X$ (see Fig. 1).

There exists a weak homotopy equivalence from the geometric realization $|\mathcal{K}(X)|$ to $X$, i.e. a continuous map $|\mathcal{K}(X)| \rightarrow X$ which induces isomorphisms in all homotopy groups. The application $\mathcal{K}$ is in fact functorial. A continuous map $f: X \rightarrow Y$ between finite $T_{0}$-spaces induces a simplicial map $\mathcal{K}(f): \mathcal{K}(X) \rightarrow \mathcal{K}(Y)$ which coincides with $f$ on vertices. Besides, it is easy to see that this construction makes the following diagram commutative

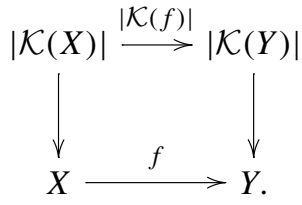

If two maps $f, g: X \rightarrow Y$ between finite $T_{0}$-spaces are homotopic, it can be proved that the simplicial maps $\mathcal{K}(f), \mathcal{K}(g): \mathcal{K}(X) \rightarrow \mathcal{K}(Y)$ lie in the same contiguity class. In particular $|\mathcal{K}(f)| \simeq|\mathcal{K}(g)|$.

The functor $\mathcal{K}$ can be used to find a simplicial complex with the same weak homotopy type of a given finite space. Recall that two spaces $X$ and $Y$ (non-necessarily finite) are said to be weak (homotopy) equivalent (or to have the same weak homotopy type) if there exists a sequence 

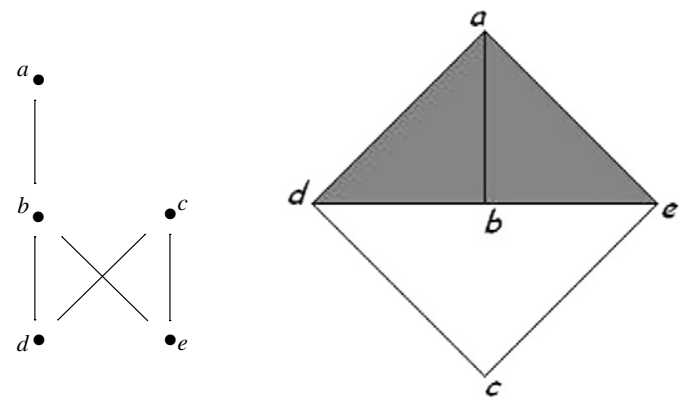

Fig. 1. A finite space and its associated simplicial complex.

of spaces $X=X_{1}, X_{2}, \ldots, X_{n}=Y$ such that for each $1 \leqslant i<n$ there is a weak homotopy equivalence $X_{i} \rightarrow X_{i+1}$ or $X_{i+1} \rightarrow X_{i}$. We will denote this by $X \stackrel{\text { we }}{\approx} Y$.

Conversely, given a finite simplicial complex $K$, one would like to find a finite model of $|K|$, i.e. a finite space which is weak equivalent to $|K|$. With this aim, McCord defined another functor, denoted by $\mathcal{X}$, that associates to each finite simplicial complex $K$ a finite $T_{0}$-space $\mathcal{X}(K)$, which is the poset of simplices of $K$ ordered by inclusion. Note that $\mathcal{K}(\mathcal{X}(K))=K^{\prime}$ is the barycentric subdivision of $K$, which implies that there exists a weak homotopy equivalence $|K| \rightarrow \mathcal{X}(K)$. The functor $\mathcal{X}$ on maps is defined as follows. Given a simplicial map $\varphi: K \rightarrow L$, we define $\mathcal{X}(\varphi): \mathcal{X}(K) \rightarrow \mathcal{X}(L)$ by $\mathcal{X}(\varphi)(S)=\varphi(S)$ for every simplex $S$ of $K$. In this case one does not have a commutative diagram as before, but a diagram that commutes up to homotopy

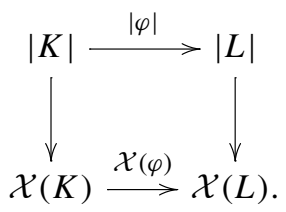

By Whitehead theorem, if $X, Y$ are finite $T_{0}$-spaces, $X \stackrel{\text { we }}{\approx} Y$ if and only if $|\mathcal{K}(X)|$ and $|\mathcal{K}(Y)|$ have the same homotopy type. On the other hand, if $K$ and $L$ are finite simplicial complexes, $|K|$ and $|L|$ are homotopy equivalent if and only if $\mathcal{X}(K) \stackrel{\text { we }}{\approx} \mathcal{X}(L)$.

\subsection{Simplicial collapses and expansions}

We finish this introductory section by recalling the basic notions on simple homotopy theory for simplicial complexes. Mainly, we want to fix the notations that we will use in Sections 3 and 4. The standard references for this are Whitehead's papers [15-17], Milnor's article [10] and M.M. Cohen's book [5].

Let $L$ be a subcomplex of a finite simplicial complex $K$. There is an elementary simplicial collapse from $K$ to $L$ if there is a simplex $S$ of $K$ and a vertex $a$ of $K$ not in $S$ such that $K=L \cup a S$ and $L \cap a S=a \dot{S}$. Here $a S$ denotes the join of $a$ and $S$ and $\dot{S}$ denotes the boundary of $S$. This is equivalent to say that there are only two simplices $S, S^{\prime}$ of $K$ which are not in $L$ and such that $S$ is a free face of $S^{\prime}$. Elementary collapses will be denoted, as usual, $K \searrow L$.

We say that $K$ (simplicially) collapses to $L$ (or that $L$ expands to $K$ ) if there exists a sequence $K=K_{1}, K_{2}, \ldots, K_{n}=L$ of finite simplicial complexes such that $K_{i} \searrow K_{i+1}$ for all $i$. This is 
denoted by $K \searrow L$ or $L \nearrow K$. Two complexes $K$ and $L$ have the same simple homotopy type if there is a sequence $K=K_{1}, K_{2}, \ldots, K_{n}=L$ such that $K_{i} \searrow K_{i+1}$ or $K_{i} \nearrow K_{i+1}$ for all $i$. Following M.M. Cohen's notation, we denote this by $K \curvearrowright L$. It is well known that $K \circlearrowleft L$ if and only if $|K|$ and $|L|$ are simple homotopy equivalent [17].

\section{Simple homotopy types: The first main theorem}

The first mathematician who investigated the relationship between finite spaces and simple homotopy types of polyhedra was T. Osaki [11]. He showed that if $x \in X$ is a beat point, $\mathcal{K}(X)$ collapses to $\mathcal{K}(X \backslash\{x\})$. In particular, if two finite $T_{0}$-spaces, $X$ and $Y$ are homotopy equivalent, their associated simplicial complexes, $\mathcal{K}(X)$ and $\mathcal{K}(Y)$, have the same simple homotopy type. However, there exist finite spaces which are not homotopy equivalent but whose associated complexes have the same simple homotopy type. Consider, for instance, the spaces with the following Hasse diagrams:
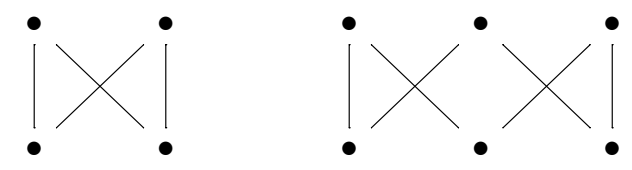

They are not homotopy equivalent because they are non-homeomorphic minimal finite spaces. However their associated complexes are triangulations of $S^{1}$ and therefore, have the same simple homotopy type.

A more interesting example is the following.

Example 3.1 (The Wallet). Let $W$ be a finite $T_{0}$-space, whose Hasse diagram is

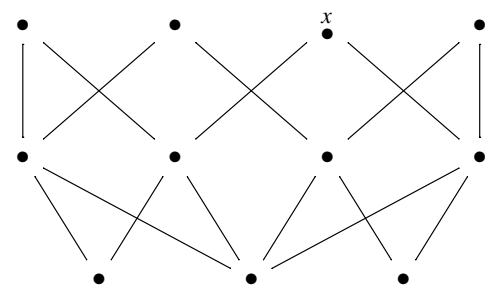

Fig. 2. $W$.

This finite space is not contractible since it does not have beat points, but it is not hard to see that $|\mathcal{K}(W)|$ is contractible and therefore, it has the same simple homotopy type as a point. In fact we will deduce from Proposition 3.3 that $W$ is a homotopically trivial space, i.e. all its homotopy groups are trivial. This example also shows that Whitehead theorem does not hold in the context of finite spaces, not even for homotopically trivial spaces.

We introduce now the notion of a weak beat point which generalizes Stong's definition of beat points. The following notations will be used in the rest of the paper. Given a point $x \in X$, we denote by $F_{x}$ the closure of $x$ in $X$, i.e. the set of points which are greater than or equal to $x$. 
We let $\hat{U}_{x}=U_{x} \backslash\{x\}$ and $\hat{F}_{x}=F_{x} \backslash\{x\}$. In case we need to specify the ambient space $X$, we will write $F_{x}^{X}, \hat{U}_{x}^{X}$ and $\hat{F}_{x}^{X}$ respectively.

Definition 3.2. Let $X$ be a finite $T_{0}$-space. We will say that $x \in X$ is a weak beat point of $X$ (or a weak point, for short) if either $\hat{U}_{x}$ is contractible or $\hat{F}_{x}$ is contractible. In the first case we say that $x$ is a down weak point and in the second, that $x$ is an up weak point.

Note that beat points are in particular weak points, for if $x \in X$ is a down beat point, $\hat{U}_{x}$ has a maximum and if $x$ is an up beat point, $\hat{F}_{x}$ has a minimum. When $x$ is a beat point of $X$, we have seen in the previous section that the inclusion $i: X \backslash\{x\} \hookrightarrow X$ is a homotopy equivalence. This is not the case if $x$ is just a weak point. However, a slightly weaker result holds.

Proposition 3.3. Let $x$ be a weak point of a finite $T_{0}$-space $X$. Then the inclusion map $i: X \backslash\{x\} \hookrightarrow X$ is a weak homotopy equivalence.

Proof. We may suppose that $x$ is a down weak point since the other case follows immediately from this one, considering $X^{\text {op }}$ instead of $X$. Note that $\mathcal{K}\left(X^{\text {op }}\right)=\mathcal{K}(X)$.

Given $y \in X$, the set $i^{-1}\left(U_{y}\right)=U_{y} \backslash\{x\}$ has a maximum if $y \neq x$ and is contractible if $y=x$. Therefore $\left.i\right|_{i^{-1}\left(U_{y}\right)}: i^{-1}\left(U_{y}\right) \rightarrow U_{y}$ is a weak homotopy equivalence for every $y \in X$. Now the result follows from Theorem 6 of [9] applied to the basis-like cover given by the minimal basis of $X$.

As an application of the last proposition, we verify that the space $W$ defined above, is a noncontractible homotopically trivial space. As we pointed out in Example 3.1, $W$ is not contractible since it is a minimal finite space with more than one point. However, it contains a weak point $x$ (see Fig. 2), since $\hat{U}_{x}$ is contractible (see Fig. 3).

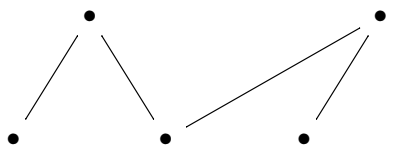

Fig. 3. $\hat{U}_{x}$.

Therefore $W$ is weak homotopy equivalent to $W \backslash\{x\}$.

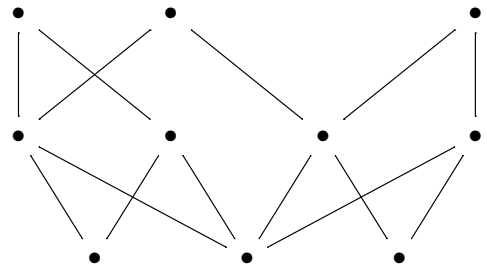

Fig. 4. $W \backslash\{x\}$.

Now it is easy to see that this subspace is contractible, because it does have beat points, and one can get rid of them one by one. 
Definition 3.4. Let $X$ be a finite $T_{0}$-space and let $Y \subsetneq X$. We say that $X$ collapses to $Y$ by an elementary collapse (or that $Y$ expands to $X$ by an elementary expansion) if $Y$ is obtained from $X$ by removing a weak point. We denote $X \searrow Y$ or $Y$ e $X$. In general, given two finite $T_{0}$-spaces $X$ and $Y$, we say that $X$ collapses to $Y$ (or $Y$ expands to $X$ ) if there is a sequence $X=X_{1}, X_{2}, \ldots, X_{n}=Y$ of finite $T_{0}$-spaces such that for each $1 \leqslant i<n, X_{i} \searrow X_{i+1}$. In this case we write $X \searrow Y$ or $Y \nearrow X$. Two finite $T_{0}$-spaces $X$ and $Y$ are simply equivalent if there is a sequence $X=X_{1}, X_{2}, \ldots, X_{n}=Y$ of finite $T_{0}$-spaces such that for each $1 \leqslant i<n, X_{i} \searrow X_{i+1}$ or $X_{i} \nearrow X_{i+1}$. We denote in this case $X \rtimes Y$, following the same notation that we adopted for simplicial complexes.

In contrast with the classical situation, where a simple homotopy equivalence is a special kind of homotopy equivalence, homotopy equivalent finite spaces are simply equivalent. It follows from Proposition 3.3 that simply equivalent finite spaces are weak equivalent.

In order to prove Theorem 3.10, we need some previous results. The first one concerns the homotopy type of the associated finite space $\mathcal{X}(K)$ of a simplicial cone $K$. Suppose $K=a L$ is a cone, i.e. $K$ is the join of a simplicial complex $L$ with a vertex $a \notin L$. Since $|K|$ is contractible, it is clear that $\mathcal{X}(K)$ is homotopically trivial. The following lemma shows that $\mathcal{X}(K)$ is in fact contractible (compare with [12]).

Lemma 3.5. Let $K=a L$ be a finite cone. Then $\mathcal{X}(K)$ is contractible.

Proof. Define $f: \mathcal{X}(K) \rightarrow \mathcal{X}(K)$ by $f(S)=S \cup\{a\}$. This function is order-preserving and therefore continuous.

If we consider the constant map $g: \mathcal{X}(K) \rightarrow \mathcal{X}(K)$ that takes all $\mathcal{X}(K)$ into $\{a\}$, we have that $1_{\mathcal{X}(K)} \leqslant f \geqslant g$. This proves that the identity is homotopic to a constant map.

The following construction is the analogue to the mapping cylinder of general spaces and the simplicial mapping cylinder of simplicial complexes.

Definition 3.6. Let $f: X \rightarrow Y$ be a map between finite $T_{0}$-spaces. We define the non-Hausdorff mapping cylinder $B(f)$ as the following finite $T_{0}$-space. The underlying set is the disjoint union $X \sqcup Y$. We keep the given ordering within $X$ and $Y$ and for $x \in X, y \in Y$ we set $x \leqslant y$ in $B(f)$ if $f(x) \leqslant y$ in $Y$.

Lemma 3.7. Let $f: X \rightarrow Y$ be a map between finite $T_{0}$-spaces such that $f^{-1}\left(U_{y}\right)$ is contractible for every $y \in Y$. Then $B(f) \searrow i(X)$ and $B(f) \searrow j(Y)$, where $i: X \hookrightarrow B(f)$ and $j: Y \hookrightarrow B(f)$ are the canonical inclusions.

Proof. Label all the elements $x_{1}, x_{2}, \ldots, x_{n}$ of $X$ in such a way that $x_{r} \leqslant x_{s}$ implies $r \leqslant s$ and define $Y_{r}=j(Y) \cup\left\{i\left(x_{1}\right), i\left(x_{2}\right), \ldots, i\left(x_{r}\right)\right\} \subseteq B(f)$ for each $0 \leqslant r \leqslant n$. Then

$$
\hat{F}_{i\left(x_{r}\right)}^{Y_{r}}=\left\{j(y) \mid y \geqslant f\left(x_{r}\right)\right\}
$$

is homeomorphic to the contractible space $F_{f\left(x_{r}\right)}^{Y}$. It follows that $Y_{r} \searrow^{e} Y_{r-1}$ for $1 \leqslant r \leqslant n$, and then $B(f)=Y_{n}$ collapses to $j(Y)=Y_{0}$. Notice that we have not yet used the fact that $f$ is distinguished. 
Now order the elements $y_{1}, y_{2}, \ldots, y_{m}$ of $Y$ in such a way that $y_{r} \leqslant y_{s}$ implies $r \leqslant s$ and define $X_{r}=i(X) \cup\left\{j\left(y_{r+1}\right), j\left(y_{r+2}\right), \ldots, j\left(y_{m}\right)\right\} \subseteq B(f)$ for every $0 \leqslant r \leqslant m$. Then

$$
\hat{U}_{j\left(y_{r}\right)}^{X_{r-1}}=\left\{i(x) \mid f(x) \leqslant y_{r}\right\}
$$

is homeomorphic to $f^{-1}\left(U_{y_{r}}\right)$, which is contractible by hypothesis. Thus $X_{r-1} \searrow X_{r}$ for $1 \leqslant$ $r \leqslant m$ and therefore $B(f)=X_{0}$ collapses to $i(X)=X_{m}$.

It is well known that any finite simplicial complex $K$ has the same simple homotopy type of its barycentric subdivision $K^{\prime}$. We prove next an analogous result for finite spaces. Following [6], the barycentric subdivision of a finite $T_{0}$-space $X$ is defined by $X^{\prime}=\mathcal{X}(\mathcal{K}(X))$. Explicitly, $X^{\prime}$ consists of the non-empty chains of $X$ ordered by inclusion. It is shown in [6] that there is a weak homotopy equivalence $h: X^{\prime} \rightarrow X$ which takes each chain $C$ to its maximum $\max (C)$.

Proposition 3.8. Let $X$ be a finite $T_{0}$-space. Then $X$ and $X^{\prime}$ are simply equivalent.

Proof. It suffices to show that the map $h: X^{\prime} \rightarrow X$ satisfies the hypothesis of Lemma 3.7. This is clear since $h^{-1}\left(U_{x}\right)=\{C \mid \max (C) \leqslant x\}=\mathcal{X}\left(\mathcal{K}\left(U_{x}\right)\right)=\mathcal{X}\left(x \mathcal{K}\left(\hat{U}_{x}\right)\right)$ is contractible by Lemma 3.5 .

Lemma 3.9. Let $L$ be a subcomplex of a finite simplicial complex $K$. Let $T$ be a set of simplices of $K$ which are not in $L$, and let a be a vertex of $K$ which is contained in no simplex of $T$, but such that a $S$ is a simplex of $K$ for every $S \in T$. Finally, suppose that $K=L \cup \bigcup_{S \in T}\{S, a S\}$ (i.e. the simplices of $K$ are those of $L$ together with the simplices $S$ and a $S$ for every $S$ in $T$ ). Then $L \nearrow K$.

Proof. Number the elements $S_{1}, S_{2}, \ldots, S_{n}$ of $T$ in such a way that for every $i, j$ with $i \leqslant j$, $\# S_{i} \leqslant \# S_{j}$. Here $\# S_{k}$ denotes the cardinality of $S_{k}$. Define $K_{i}=L \cup \bigcup_{j=1}^{i}\left\{S_{j}, a S_{j}\right\}$ for $0 \leqslant i \leqslant n$. Let $S \subsetneq S_{i}$. If $S \in T$, then $S, a S \in K_{i-1}$, since \#S<\#S . If $S \notin T$, then $S, a S \in L \subseteq K_{i-1}$. This proves that $a S_{i} \cap K_{i-1}=a \dot{S}_{i}$.

By induction, $K_{i}$ is a simplicial complex for every $i$, and $K_{i-1}$ e/ $K_{i}$. Therefore $L=$ $K_{0} \nearrow K_{n}=K$.

Now we are ready to prove the first main result of this article.

\section{Theorem 3.10.}

(a) Let $X$ and $Y$ be finite $T_{0}$-spaces. Then, $X$ and $Y$ are simply equivalent if and only if $\mathcal{K}(X)$ and $\mathcal{K}(Y)$ have the same simple homotopy type. Moreover, if $X \searrow Y$ then $\mathcal{K}(X) \searrow \mathcal{K}(Y)$.

(b) Let $K$ and $L$ be finite simplicial complexes. Then, $K$ and $L$ are simple homotopy equivalent if and only if $\mathcal{X}(K)$ and $\mathcal{X}(L)$ are simply equivalent. Moreover, if $K \searrow L$ then $\mathcal{X}(K) \searrow \mathcal{X}(L)$.

Proof. Let $X$ be a finite $T_{0}$-space and let $x \in X$ be a weak point. We will show first that $\mathcal{K}(X \backslash\{x\}) \nearrow \mathcal{K}(X)$. We may suppose that $x$ is a down weak point since the other case follows immediately from this one replacing $X$ by $X^{\mathrm{op}}$. Since $\hat{U}_{x}$ is contractible, there exists a sequence of spaces $\hat{U}_{x}=X_{n} \supsetneq X_{n-1} \supsetneq \cdots \supsetneq X_{1}=\left\{x_{1}\right\}$, with $X_{i}=\left\{x_{1}, x_{2}, \ldots, x_{i}\right\}$ and such that $x_{i}$ is a 
beat point of $X_{i}$ for each $i \geqslant 2$. By Remark 2.2, it follows that there exists $y_{i} \in X_{i-1}$ for each $2 \leqslant i \leqslant n$ with the following property: if $z \in X_{i}$ is comparable with $x_{i}$, then it is comparable with $y_{i}$.

Let $K_{i} \subseteq \mathcal{K}(X)$ be the subcomplex whose simplices are the chains of $X \backslash\{x\}$ together with the chains of $F_{x} \cup X_{i} \subseteq X$. In other words, $K_{i}=\mathcal{K}(X \backslash\{x\}) \cup \mathcal{K}\left(F_{X} \cup X_{i}\right)$. We will prove that $\mathcal{K}(X \backslash\{x\}) \nearrow K_{1} \nearrow K_{2} \nearrow \cdots \nearrow K_{n}=\mathcal{K}(X)$.

In order to prove that $\mathcal{K}(X \backslash\{x\}) \nearrow K_{1}$, we apply Lemma 3.9 with $L=\mathcal{K}(X \backslash\{x\}), K=K_{1}$, $T=\left\{S \in K_{1} \mid x \in S, x_{1} \notin S\right\}$ and $a=x_{1}$. Note that $x_{1} S \in K_{1}$ for every $S \in T$ since any element of $S$ is greater than or equal to $x$ and therefore, comparable with $x_{1}$. In order to see that $K_{i-1} \nearrow K_{i}$ for $i \geqslant 2$, note that the simplices of $K_{i}$ which are not in $K_{i-1}$ are the chains of $F_{x} \cup X_{i}$ that contain both $x$ and $x_{i}$. We apply again Lemma 3.9 with $L=K_{i-1}, K=K_{i}$, $T=\left\{S \in K_{i} \mid x, x_{i} \in S, y_{i} \notin S\right\}$ and $a=y_{i}$. Note that if $S \in T$ and $y \in S$, then either $y \in X_{i}$ and it is comparable with $x_{i}$ or $y \geqslant x$. In any of these cases $y$ is comparable with $y_{i}$, and therefore $y_{i} S \in K_{i}$. We have then proved that $X \searrow Y$ implies $\mathcal{K}(X) \searrow \mathcal{K}(Y)$. In particular, $X \searrow Y$ implies $\mathcal{K}(X) \curvearrowright \mathcal{K}(Y)$.

Suppose now that $K$ and $L$ are finite simplicial complexes such that $K \searrow L$. Then, there exist $S \in K$ and a vertex $a$ of $K$ not in $S$ such that $a S \in K, K=L \cup\{S, a S\}$ and $a S \cap L=a \dot{S}$. It follows that $S$ is an up beat point of $\mathcal{X}(K)$, and since $\hat{U}_{a S}^{\mathcal{X}(K) \backslash\{S\}}=\mathcal{X}(a \dot{S})$, by Lemma 3.5, aS is a down weak point of $\mathcal{X}(K) \backslash\{S\}$. Therefore $\mathcal{X}(K) \searrow \mathcal{X}(K) \backslash\{S\} \searrow \mathcal{X}(K) \backslash\{S, a S\}=$ $\mathcal{X}(L)$. This proves the first part of (b) and the "moreover" part.

Let $X, Y$ be finite $T_{0}$-spaces such that $\mathcal{K}(X) \curvearrowright \mathcal{K}(Y)$. Then $X^{\prime}=\mathcal{X}(\mathcal{K}(X)) \curvearrowright \mathcal{X}(\mathcal{K}(Y))=$ $Y^{\prime}$ and by Proposition 3.8, $X \rtimes Y$. Finally, if $K, L$ are finite simplicial complexes such that $\mathcal{X}(K) \bigcirc \mathcal{X}(L), K^{\prime}=\mathcal{K}(\mathcal{X}(K)) \curvearrowright \mathcal{K}(\mathcal{X}(L))=L^{\prime}$ and therefore $K \curvearrowright L$. This completes the proof.

Corollary 3.11. The functors $\mathcal{K}, \mathcal{X}$ induce a one-to-one correspondence between simple equivalence classes of finite spaces and simple homotopy types of finite simplicial complexes

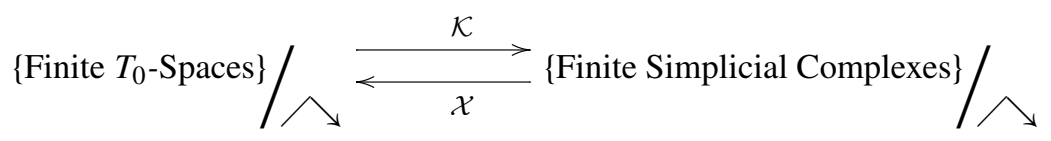

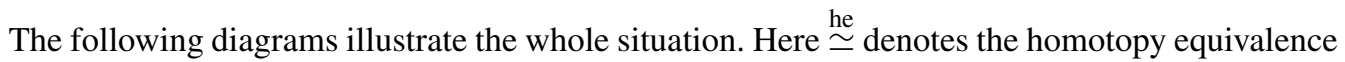
relation.

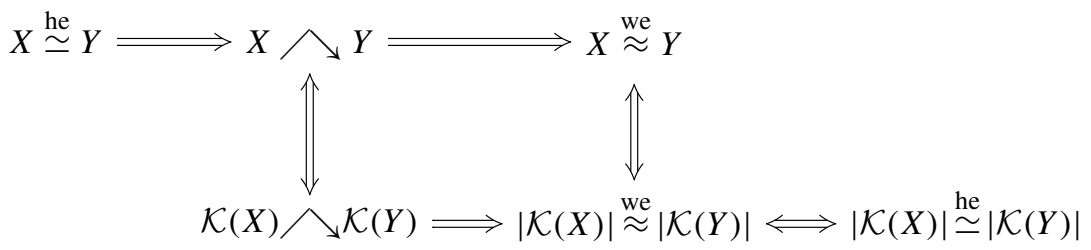




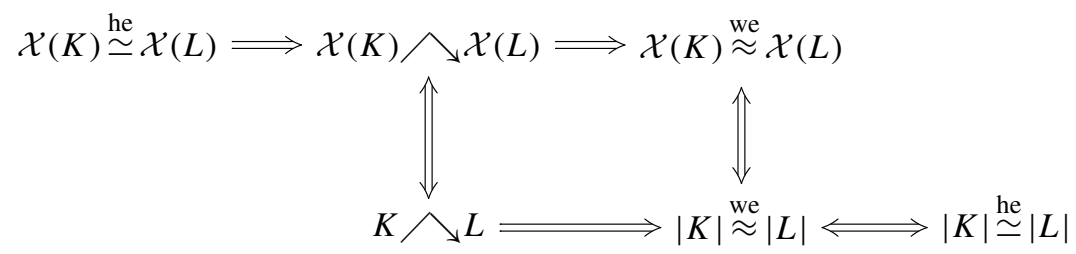

The Wallet $W$ satisfies $W \searrow *$, however $W \not$ he $*$. Therefore $X \curvearrowright Y \nRightarrow X \cong Y$ he Since $|K| \stackrel{\text { he }}{\simeq}|L| \nRightarrow K \curvearrowright L, X \stackrel{\text { we }}{\approx} Y \nRightarrow X \searrow Y$. Note that, if $X \stackrel{\text { we }}{\approx} Y$ and their Whitehead group $W h\left(\pi_{1}(X)\right)$ is trivial, then $|\mathcal{K}(X)|$ and $|\mathcal{K}(Y)|$ are simple homotopy equivalent CW-complexes. It follows from Theorem 3.10 that $X \curvearrowright Y$. Thus, we have proved

Corollary 3.12. Let $X, Y$ be weak equivalent finite $T_{0}$-spaces such that $W h\left(\pi_{1}(X)\right)=0$. Then $X \curvearrowright Y$.

Another immediate consequence of the theorem is the following

Corollary 3.13. Let $X, Y$ be finite $T_{0}$-spaces. If $X \searrow Y$, then $X^{\prime} \searrow Y^{\prime}$.

Note that from Theorem 3.10 one also deduces the following well-known fact: If $K$ and $L$ are finite simplicial complexes such that $K \searrow L$, then $K^{\prime} \searrow L^{\prime}$.

One of the most important open results concerning collapsible complexes is Zeeman's Conjecture [18], which states that if $K$ is a contractible polyhedron of dimension 2, $K \times I$ is collapsible. This conjecture implies the 3-dimensional Poincaré Conjecture (see [18]). The notion of collapsibility for finite spaces is closely related with the analogous notion for simplicial complexes: We say that a finite $T_{0}$-space is collapsible if it collapses to a point. Observe that every contractible finite $T_{0}$-space is collapsible, however the converse is not true. The Wallet $W$ introduced in Example 3.1 is collapsible and non-contractible. Note that if a finite $T_{0}$-space $X$ is collapsible, its associated simplicial complex $\mathcal{K}(X)$ is also collapsible. Moreover, if $K$ is a collapsible complex, then $\mathcal{X}(K)$ is a collapsible finite space. Therefore, if $X$ is a collapsible finite space, its subdivision $X^{\prime}$ is also collapsible.

Let us consider now a compact contractible polyhedron $X$ with the property that any triangulation of $X$ is non-collapsible, for instance the Dunce Hat [18]. Let $K$ be any triangulation of $X$. The associated finite space $\mathcal{X}(K)$ is homotopically trivial because $X$ is contractible. However, $\mathcal{X}(K)$ is not collapsible since $K^{\prime}$ is not collapsible.

We have therefore the following strict implications in the context of finite spaces:

$$
\text { contractible } \Rightarrow \text { collapsible } \Rightarrow \text { homotopically trivial. }
$$

As we pointed out in the previous section, the beat points defined by Stong provide an effective way of deciding whether two finite spaces are homotopy equivalent. The problem becomes much harder when one deals with weak homotopy types instead. In [2] we have studied the minimal finite models of a given space $X$, which are the smallest spaces weak equivalent to $X$. In that article we characterized the minimal finite models of spheres and finite graphs (finite CWcomplexes of dimension one). We proved that, in general, the minimal finite models of a space 
are not unique. For example $\bigvee_{i=1}^{3} S^{1}$ has three minimal finite models up to homeomorphism. It makes sense to formulate the following definition.

Definition 3.14. A minimal simple model of a finite $T_{0}$-space $X$ is a finite $T_{0}$-space simply equivalent to $X$ of minimum cardinality. We will say that a space is a minimal simple model if it is a minimal simple model of itself.

Clearly, one has the following implications:

$$
\text { minimal finite model } \Rightarrow \text { minimal simple model } \Rightarrow \text { minimal finite space. }
$$

Note that if the Whitehead group $W h\left(\pi_{1}(X)\right)$ is trivial, the converse of the first implication holds. Therefore, given a finite $T_{0}$-space $X$ such that $W h\left(\pi_{1}(X)\right)=0$, one could reach any minimal finite model of $X$ just by adding and removing weak points from $X$. Elementary collapses and expansions provide a tool of reduction when the space has trivial Whitehead group. Unfortunately it is not always possible to obtain a minimal simple model by only removing weak points. For example, take any homotopically trivial non-collapsible finite space.

Of course there is not uniqueness of minimal simple models. Consider for instance the space $\mathbb{S} D_{3}$

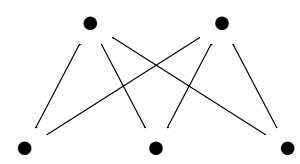

and its opposite, which are minimal simple models because they are minimal finite models. Notice that $\mathbb{S} D_{3} \frown\left(\mathbb{S} D_{3}\right)^{\text {op }}$ and they are not homeomorphic.

\section{Simple homotopy equivalences: The second main theorem}

In this section we prove the second main result of the article, which relates simple homotopy equivalences of complexes with simple equivalences between finite spaces. Like in the classical setting, the class of simple equivalences is generated by the elementary expansions. However, in the context of finite spaces this class is also generated by the distinguished maps, which play a key role in this theory.

Recall that a homotopy equivalence $f:|K| \rightarrow|L|$ between compact polyhedra is a simple homotopy equivalence if it is homotopic to a composition of a finite sequence of maps $|K| \rightarrow$ $\left|K_{1}\right| \rightarrow \cdots \rightarrow\left|K_{n}\right| \rightarrow|L|$, each of them an expansion or a homotopy inverse of one $[5,13]$.

We prove first that homotopy equivalences between finite spaces induce simple homotopy equivalences between the associated polyhedra.

Theorem 4.1. If $f: X \rightarrow Y$ is a homotopy equivalence between finite $T_{0}$-spaces, then $|\mathcal{K}(f)|:|\mathcal{K}(X)| \rightarrow|\mathcal{K}(Y)|$ is a simple homotopy equivalence.

Proof. Let $X_{c}$ and $Y_{c}$ be cores of $X$ and $Y$. Let $i_{X}: X_{c} \rightarrow X$ and $i_{Y}: Y_{c} \rightarrow Y$ be the inclusions and $r_{X}: X \rightarrow X_{c}, r_{Y}: Y \rightarrow Y_{c}$ retractions of $i_{X}$ and $i_{Y}$ such that $i_{X} r_{X} \simeq 1_{X}$ and $i_{Y} r_{Y} \simeq 1_{Y}$. 
Since $r_{Y} f i_{X}: X_{c} \rightarrow Y_{c}$ is a homotopy equivalence between minimal finite spaces, it is a homeomorphism. Therefore $\mathcal{K}\left(r_{Y} f i_{X}\right): \mathcal{K}\left(X_{c}\right) \rightarrow \mathcal{K}\left(Y_{c}\right)$ is an isomorphism and then $\left|\mathcal{K}\left(r_{Y} f i_{X}\right)\right|$ is a simple homotopy equivalence. Since $\mathcal{K}(X) \searrow \mathcal{K}\left(X_{c}\right),\left|\mathcal{K}\left(i_{X}\right)\right|$ is a simple homotopy equivalence, and then the homotopy inverse $\left|\mathcal{K}\left(r_{X}\right)\right|$ is also a simple homotopy equivalence. Analogously $\left|\mathcal{K}\left(i_{Y}\right)\right|$ is a simple homotopy equivalence.

Finally, since $f \simeq i_{Y} r_{Y} f i_{X} r_{X}$, it follows that $|\mathcal{K}(f)| \simeq\left|\mathcal{K}\left(i_{Y}\right)\right|\left|\mathcal{K}\left(r_{Y} f i_{X}\right)\right|\left|\mathcal{K}\left(r_{X}\right)\right|$ is a simple homotopy equivalence.

In order to describe the class of simple equivalences, we will use a kind of maps that was already studied in Lemma 3.7.

Definition 4.2. A map $f: X \rightarrow Y$ between finite $T_{0}$-spaces is distinguished if $f^{-1}\left(U_{y}\right)$ is contractible for each $y \in Y$. We denote by $\mathcal{D}$ the class of distinguished maps.

Note that by the theorem of McCord [9, Theorem 6], every distinguished map is a weak homotopy equivalence and therefore induces a homotopy equivalence between the associated complexes. We will prove in Theorem 4.4 that in fact the induced map is a simple homotopy equivalence. From the proof of Proposition 3.3, it is clear that if $x \in X$ is a down weak point, the inclusion $X \backslash\{x\} \hookrightarrow X$ is distinguished.

Remark 4.3. The map $h: X^{\prime} \rightarrow X$ defined by $h(C)=\max (C)$, is distinguished by the proof of Proposition 3.8 .

Clearly, homeomorphisms are distinguished. However it is not difficult to show that homotopy equivalences are not distinguished in general.

Theorem 4.4. Every distinguished map induces a simple homotopy equivalence.

Proof. Suppose $f: X \rightarrow Y$ is distinguished. Consider the non-Hausdorff mapping cylinder $B(f)$ and the canonical inclusions $i: X \hookrightarrow B(f), j: Y \hookrightarrow B(f)$.

The following diagram

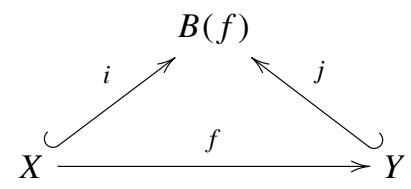

does not commute, but $i \leqslant j f$ and then $i \simeq j f$. Therefore $|\mathcal{K}(i)| \simeq|\mathcal{K}(j)||\mathcal{K}(f)|$. By Lemma 3.7 and Theorem 3.10, $|\mathcal{K}(i)|$ and $|\mathcal{K}(j)|$ are expansions (composed with isomorphisms) and then, $|\mathcal{K}(f)|$ is a simple homotopy equivalence.

We have already shown that expansions, homotopy equivalences and distinguished maps induce simple homotopy equivalences at the level of complexes. Note that if $f, g, h$ are three maps between finite $T_{0}$-spaces such that $f g \simeq h$ and two of them induce simple homotopy equivalences, then so does the third. 
Definition 4.5. Let $\mathcal{C}$ be a class of continuous maps between topological spaces. We say that $\mathcal{C}$ is closed if it satisfies the following homotopy 2-out-of-3 property: For any $f, g, h$ with $f g \simeq h$, if two of the three maps are in $\mathcal{C}$, then so is the third.

Definition 4.6. Let $\mathcal{C}$ be a class of continuous maps. The class $\overline{\mathcal{C}}$ generated by $\mathcal{C}$ is the smallest closed class containing $\mathcal{C}$.

It is clear that $\overline{\mathcal{C}}$ is always closed under composition and homotopy. The class of simple homotopy equivalences between $\mathrm{CW}$-complexes is closed and it is generated by the elementary expansions. Note that every map in the class $\mathcal{E}$ of elementary expansions between finite spaces induces a simple homotopy equivalence at the level of complexes and therefore the same holds for the maps of $\overline{\mathcal{E}}$. Contrary to the case of CW-complexes, a map between finite spaces which induces a simple homotopy equivalence, need not have a homotopy inverse. This is the reason why the definition of $\overline{\mathcal{E}}$ is not as simple as in the setting of complexes. We will prove that $\overline{\mathcal{E}}=\overline{\mathcal{D}}$, the class generated by the distinguished maps.

A map $f: X \rightarrow Y$ such that $f^{-1}\left(F_{y}\right)$ is contractible for every $y$, need not be distinguished. However we will show that $f \in \overline{\mathcal{D}}$. We denote by $f^{\text {op }}: X^{\text {op }} \rightarrow Y^{\text {op }}$ the map that coincides with $f$ in the underlying sets, and let $\mathcal{D}^{\text {op }}=\left\{f \mid f^{\text {op }} \in \mathcal{D}\right\}$.

Lemma 4.7. $\overline{\mathcal{D}^{\mathrm{op}}}=\overline{\mathcal{D}}$.

Proof. Suppose that $f: X \rightarrow Y$ lies in $\mathcal{D}^{\text {op }}$. Consider the following commutative diagram

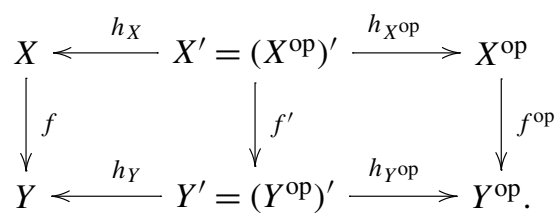

Here, $f^{\prime}$ denotes the map $\mathcal{X}(\mathcal{K}(f))$. Since $\overline{\mathcal{D}}$ satisfies the 2 -out-of-3 property and $h_{X^{\text {op }}}, h_{Y_{\text {op }}}$, $f^{\text {op }}$ are distinguished by Remark 4.3, $f^{\prime} \in \overline{\mathcal{D}}$. And since $h_{X}, h_{Y}$ are distinguished, $f \in \overline{\mathcal{D}}$. This proves that $\overline{\mathcal{D}^{\text {op }}} \subseteq \overline{\mathcal{D}}$. The other inclusion follows analogously from the opposite diagram.

Proposition 4.8. $\overline{\mathcal{E}}=\overline{\mathcal{D}}$, and this class contains all homotopy equivalences between finite $T_{0^{-}}$ spaces.

Proof. Every expansion of finite spaces is in $\overline{\mathcal{E}}$ because it is a composition of maps in $\mathcal{E}$.

Let $f: X \rightarrow Y$ be distinguished. By the proof of Theorem 4.4 there exist expansions (eventually composed with homeomorphisms) $i, j$, such that $i \simeq j f$. Therefore $f \in \overline{\mathcal{E}}$.

If $x \in X$ is a down weak point, the inclusion $X \backslash\{x\} \hookrightarrow X$ is distinguished. If $x$ is an up weak point, $X \backslash\{x\} \hookrightarrow X$ lies in $\overline{\mathcal{D}}$ by the previous lemma and therefore $\overline{\mathcal{E}} \subseteq \overline{\mathcal{D}}$.

Suppose now that $f: X \rightarrow Y$ is a homotopy equivalence. From the proof of Theorem 4.1, $f i_{X} \simeq i_{Y} r_{Y} f i_{X}$ where $i_{X}, i_{Y}$ are expansions and $r_{Y} f i_{X}$ is a homeomorphism. This implies that $f \in \overline{\mathcal{E}}=\overline{\mathcal{D}}$. 
We denote by $\mathcal{S}=\overline{\mathcal{E}}=\overline{\mathcal{D}}$ the class of simple equivalences between finite spaces. In the rest of the paper we study the relationship between simple equivalences of finite spaces and simple homotopy equivalences of polyhedra.

Lemma 4.9. Let $\varphi, \psi: K \rightarrow L$ be simplicial maps which lie in the same contiguity class. Then $\mathcal{X}(\varphi) \simeq \mathcal{X}(\psi)$.

Proof. Assume that $\varphi$ and $\psi$ are contiguous. Then the map $f: \mathcal{X}(K) \rightarrow \mathcal{X}(L)$, defined by $f(S)=\varphi(S) \cup \psi(S)$ is well-defined and continuous. Moreover $\mathcal{X}(\varphi) \leqslant f \geqslant \mathcal{X}(\psi)$, and then $\mathcal{X}(\varphi) \simeq \mathcal{X}(\psi)$.

Given $n \in \mathbb{N}$ we denote by $K^{n}$ the $n$th barycentric subdivision of $K$.

Lemma 4.10. Let $\lambda: K^{n} \rightarrow K$ be a simplicial approximation to the identity. Then $\mathcal{X}(\lambda) \in \mathcal{S}$.

Proof. It suffices to prove the case $n=1$. Suppose $\lambda: K^{\prime} \rightarrow K$ is a simplicial approximation of $1_{|K|}$. Then $\mathcal{X}(\lambda): \mathcal{X}(K)^{\prime} \rightarrow \mathcal{X}(K)$ is homotopic to $h_{\mathcal{X}(K)}$, for if $S_{1} \subsetneq S_{2} \subsetneq \cdots \subsetneq S_{m}$ is a chain of simplices of $K$, then $\mathcal{X}(\lambda)\left(\left\{S_{1}, S_{2}, \ldots, S_{m}\right\}\right)=\left\{\lambda\left(S_{1}\right), \lambda\left(S_{2}\right), \ldots, \lambda\left(S_{m}\right)\right\} \subseteq S_{m}=$ $h_{\mathcal{X}(K)}\left(\left\{S_{1}, S_{2}, \ldots, S_{m}\right\}\right)$. By Remark 4.3, it follows that $\mathcal{X}(\lambda) \in \mathcal{S}$.

Lemma 4.11. Let $\varphi, \psi: K \rightarrow L$ be simplicial maps such that $|\varphi| \simeq|\psi|$. If $\mathcal{X}(\varphi) \in \mathcal{S}$, then $\mathcal{X}(\psi)$ also lies in $\mathcal{S}$.

Proof. There exists an approximation to the identity $\lambda: K^{n} \rightarrow K$ for some $n \geqslant 1$, such that $\varphi \lambda$ and $\psi \lambda$ lie in the same contiguity class. By Lemma 4.9, $\mathcal{X}(\varphi) \mathcal{X}(\lambda)=\mathcal{X}(\varphi \lambda) \simeq \mathcal{X}(\psi \lambda)=$ $\mathcal{X}(\psi) \mathcal{X}(\lambda)$. By Lemma 4.10, $\mathcal{X}(\lambda) \in \mathcal{S}$ and since $\mathcal{X}(\varphi) \in \mathcal{S}$, it follows that $\mathcal{X}(\psi) \in \mathcal{S}$.

Theorem 4.12. Let $K_{0}, K_{1}, \ldots, K_{n}$ be finite simplicial complexes and let

$$
\left|K_{0}\right| \stackrel{f_{0}}{\longrightarrow}\left|K_{1}\right| \stackrel{f_{1}}{\longrightarrow} \cdots \stackrel{f_{n-1}}{\longrightarrow}\left|K_{n}\right|
$$

be a sequence of continuous maps such that for each $0 \leqslant i<n$ either

(1) $f_{i}=\left|\varphi_{i}\right|$ where $\varphi_{i}: K_{i} \rightarrow K_{i+1}$ is a simplicial map such that $\mathcal{X}\left(\varphi_{i}\right) \in \mathcal{S}$ or

(2) $f_{i}$ is a homotopy inverse of a map $\left|\varphi_{i}\right|$ with $\varphi_{i}: K_{i+1} \rightarrow K_{i}$ a simplicial map such that $\mathcal{X}\left(\varphi_{i}\right) \in \mathcal{S}$.

If $\varphi: K_{0} \rightarrow K_{n}$ is a simplicial map such that $|\varphi| \simeq f_{n-1} f_{n-2} \ldots f_{0}$, then $\mathcal{X}(\varphi) \in \mathcal{S}$.

Proof. We may assume that $f_{0}$ satisfies condition (1). Otherwise we define $\widetilde{K}_{0}=K_{0}, \widetilde{f}_{0}=$ $\left|1_{K_{0}}\right|:\left|\widetilde{K_{0}}\right| \rightarrow\left|K_{0}\right|$ and then $|\varphi| \simeq f_{n-1} f_{n-2} \ldots f_{0} \widetilde{f}_{0}$.

We proceed by induction on $n$. If $n=1,|\varphi| \simeq\left|\varphi_{0}\right|$ where $\mathcal{X}\left(\varphi_{0}\right) \in \mathcal{S}$ and the result follows from Lemma 4.11. Suppose now that $n \geqslant 1$ and let $K_{0}, K_{1}, \ldots, K_{n}, K_{n+1}$ be finite simplicial complexes and $f_{i}:\left|K_{i}\right| \rightarrow\left|K_{i+1}\right|$ maps satisfying conditions (1) or (2), $f_{0}$ satisfying condition (1). Let $\varphi: K_{0} \rightarrow K_{n+1}$ be a simplicial map such that $|\varphi| \simeq f_{n} f_{n-1} \ldots f_{0}$. We consider two cases: $f_{n}$ satisfies condition (1) or $f_{n}$ satisfies condition (2). 
In the first case we define $g:\left|K_{0}\right| \rightarrow\left|K_{n}\right|$ by $g=f_{n-1} f_{n-2} \ldots f_{0}$. Let $\tilde{g}: K_{0}^{m} \rightarrow K_{n}$ be a simplicial approximation to $g$ and let $\lambda: K_{0}^{m} \rightarrow K_{0}$ be a simplicial approximation to the identity. Then $|\tilde{g}| \simeq g|\lambda|=f_{n-1} f_{n-2} \ldots f_{1}\left(f_{0}|\lambda|\right)$ where $f_{0}|\lambda|=\left|\varphi_{0} \lambda\right|$ and $\mathcal{X}\left(\varphi_{0} \lambda\right)=\mathcal{X}\left(\varphi_{0}\right) \mathcal{X}(\lambda) \in \mathcal{S}$ by Lemma 4.10. By induction, $\mathcal{X}(\tilde{g}) \in \mathcal{S}$, and then $\mathcal{X}\left(\varphi_{n} \tilde{g}\right) \in \mathcal{S}$. Since $|\varphi \lambda| \simeq f_{n} g|\lambda| \simeq f_{n}|\tilde{g}|=$ $\left|\varphi_{n} \tilde{g}\right|$, by Lemma $4.11, \mathcal{X}(\varphi \lambda)$ lies in $\mathcal{S}$. Therefore $\mathcal{X}(\varphi) \in \mathcal{S}$.

In the other case, $\left|\varphi_{n} \varphi\right| \simeq f_{n-1} f_{n-2} \ldots f_{0}$ and by induction, $\mathcal{X}\left(\varphi_{n} \varphi\right) \in \mathcal{S}$. Therefore $\mathcal{X}(\varphi)$ also lies in $\mathcal{S}$.

\section{Theorem 4.13.}

(a) Let $f: X \rightarrow Y$ be a map between finite $T_{0}$-spaces. Then $f$ is a simple equivalence if and only if $|\mathcal{K}(f)|:|\mathcal{K}(X)| \rightarrow|\mathcal{K}(Y)|$ is a simple homotopy equivalence.

(b) Let $\varphi: K \rightarrow L$ be a simplicial map between finite simplicial complexes. Then $|\varphi|$ is a simple homotopy equivalence if and only if $\mathcal{X}(\varphi)$ is a simple equivalence.

Proof. By definition, if $f \in \mathcal{S},|\mathcal{K}(f)|$ is a simple homotopy equivalence.

Let $\varphi: K \rightarrow L$ be a simplicial map such that $|\varphi|$ is a simple homotopy equivalence. Then there exist finite complexes $K=K_{0}, K_{1}, \ldots, K_{n}=L$ and maps $f_{i}:\left|K_{i}\right| \rightarrow\left|K_{i+1}\right|$, which are simplicial expansions or homotopy inverses of simplicial expansions, and such that $|\varphi| \simeq$ $f_{n-1} f_{n-2} \ldots f_{0}$. By Theorem 3.10, simplicial expansions between complexes induce expansions between the associated finite spaces and therefore, by Theorem $4.12, \mathcal{X}(\varphi) \in \mathcal{S}$.

Suppose now that $f: X \rightarrow Y$ is a map such that $|\mathcal{K}(f)|$ is a simple homotopy equivalence. Then, $f^{\prime}=\mathcal{X}(\mathcal{K}(f)): X^{\prime} \rightarrow Y^{\prime}$ lies in $\mathcal{S}$. Since $f h_{X}=h_{Y} f^{\prime}, f \in \mathcal{S}$.

Finally, if $\varphi: K \rightarrow L$ is a simplicial map such that $\mathcal{X}(\varphi) \in \mathcal{S},\left|\varphi^{\prime}\right|:\left|K^{\prime}\right| \rightarrow\left|L^{\prime}\right|$ is a simple homotopy equivalence. Here $\varphi^{\prime}=\mathcal{K}(\mathcal{X}(\varphi))$ is the barycentric subdivision of $\varphi$. Let $\lambda_{K}: K^{\prime} \rightarrow$ $K$ and $\lambda_{L}: L^{\prime} \rightarrow L$ be simplicial approximations to the identities. Then $\lambda_{L} \varphi^{\prime}$ and $\varphi \lambda_{K}$ are contiguous. In particular $\left|\lambda_{L}\right|\left|\varphi^{\prime}\right| \simeq|\varphi|\left|\lambda_{K}\right|$ and then $|\varphi|$ is a simple homotopy equivalence.

In the setting of finite spaces one has the following strict inclusions

\{homotopy equivalences $\} \subsetneq \mathcal{S} \subsetneq\{$ weak equivalences $\}$.

Clearly, if $f: X \rightarrow Y$ is a weak homotopy equivalence between finite $T_{0}$-spaces with trivial Whitehead group, $f \in \mathcal{S}$.

\section{References}

[1] P.S. Alexandroff, Diskrete Räume, Math. Sb. (N.S.) 2 (1937) 501-518.

[2] J.A. Barmak, E.G. Minian, Minimal finite models, J. Homotopy Relat. Struct. 2 (2007) 127-140.

[3] A. Björner, Nerves, fibers and homotopy groups, J. Combin. Theory Ser. A 102 (2003) 88-93.

[4] A. Björner, M. Wachs, V. Welker, Poset fiber theorems, Trans. Amer. Math. Soc. 357 (2004) 1877-1899.

[5] M.M. Cohen, A Course in Simple Homotopy Theory, Springer-Verlag, New York, 1970.

[6] K.A. Hardie, J.J.C. Vermeulen, Homotopy theory of finite and locally finite $T_{0}$-spaces, Exp. Math. 11 (1993) 331341.

[7] J.P. May, Finite topological spaces, Notes for REU (2003). Available at http://www.math.uchicago.edu/ may/ MISCMaster.html.

[8] J.P. May, Finite spaces and simplicial complexes, Notes for REU (2003). Available at http://www.math.uchicago. edu/ may/MISCMaster.html. 
[9] M.C. McCord, Singular homology groups and homotopy groups of finite topological spaces, Duke Math. J. 33 (1966) 465-474.

[10] J. Milnor, Whitehead Torsion, Bull. Amer. Math. Soc. 72 (1966) 358-426.

[11] T. Osaki, Reduction of finite topological spaces, Interdiscip. Inform. Sci. 5 (1999) 149-155.

[12] D. Quillen, Homotopy properties of the poset of non-trivial p-subgroups of a group, Adv. Math. 28 (1978) 101-128.

[13] L.C. Siebenmann, Infinite simple homotopy types, Indag. Math. 32 (1970) 479-495.

[14] R.E. Stong, Finite topological spaces, Trans. Amer. Math. Soc. 123 (1966) 325-340.

[15] J.H. C Whitehead, Simplicial spaces, nuclei and $m$-groups, Proc. London Math. Soc. 45 (1939) $243-327$.

[16] J.H. C Whitehead, On incidence matrices, nuclei and homotopy types, Ann. of Math. 42 (1941) 1197-1239.

[17] J.H. C Whitehead, Simple homotopy types, Amer. J. Math. 72 (1950) 1-57.

[18] E.C. Zeeman, On the dunce hat, Topology 2 (1964) 341-358. 